\title{
Hypoxia Pre-Challenged Glioma-Derived Exosomes Promote Recovery From Spinal Cord Injury by Inducing M2 Macrophage Polarization via Modulating MicroRNA-1246/TERF2IP and Let- 7b/TERF2IP Signaling Pathway
}

\section{Zhongzheng Zhi}

Shanghai Fourth People's Hospital affiliated to Tongji University School of Medicine

Chenglin Zhang

Shanghai Jiaotong University

\section{Yingjie Wang}

Shanghai Fourth People's Hospital affiliated to Tongji University School of Medicine Jian Kang

Shanghai Fourth People's Hospital affiliated to Tongji University School of Medicine

Wei Yuan

Shanghai Fourth People's Hospital affiliated to Tongji University School of Medicine Jingdong Liu

Shanghai Fourth People's Hospital affiliated to Tongji University School of Medicine

Furong Wu

Shanghai Jiaotong University

Guanghui Xu ( $\sim$ guanghuixu008@yeah.net)

Shanghai Fourth People's Hospital affiliated to Tongji University School of Medicine

\section{Research Article}

Keywords: Spinal cord injury, Glioma-derived exosomes, hypoxia, miR-1246, let-7b, TERF2IP

Posted Date: May 19th, 2021

DOI: https://doi.org/10.21203/rs.3.rs-530075/v1

License: (c) (i) This work is licensed under a Creative Commons Attribution 4.0 International License. Read Full License 


\section{Abstract}

Background: Hypoxic GLIOMA derived exosomes may induce M2 macrophage polarization by upregulating TERF2IP expression. Furthermore, M2 macrophage polarization was found to be associated with accelerated $\mathrm{SCl}$ recovery by suppressing inflammatory response. The underlying mechanism of the therapeutic role of hypoxic GLIOMA derived exosomes in $\mathrm{SCl}$ recovery remains to be explored.

Methods: Electron microscopy and Western blot were used to characterize U251 derived exosomes. Quantitative real-time PCR was performed to measure the mRNA expression of target genes, and Western blot and IHC were used to evaluate the protein expression of target genes. ELISA was performed to examine the levels of cytokines. Luciferase assay was carried out to explore the inhibitory role of miR$1246 /$ let-7b in the expression of TERF2IP. TUNEL was performed to evaluate the apoptosis of spinal cord cells in SCl rats.

Results: Hypoxic U251 derived exosomes significantly enhanced the expression of CD163, IL-10, IL-1RA, TGFB1, and CCL2 as well as the proportion of CD11b+/CD163+ cells while suppressing the expression of TNFa in U937 cells. Furthermore, the expression of miR-1246 and let-7b was remarkably elevated by Hypoxic U251 derived exosomes, while the expression of TERF2IP was inhibited. Luciferase assay demonstrated that miR-1246/let-7b effectively suppressed the expression of TERF2IP through binding to its 3' UTR. In an SCl rat model, hypoxic U251 derived exosomes notably promoted the survival and functional recovery of left hindlimb by up-regulating IL-10, miR-1246, and let-7b expression while downregulating TNFa/TERF2IP expression and attenuating apoptosis of spinal cord cells.

Conclusion: The findings of this study demonstrated that glioma derived exosomes upregulated the expression of miR-1246 and let-7b to suppress the expression of TERF2IP to induce M2 macrophage polarization. The promoted M2 macrophage polarization suppressed inflammatory response to accelerate the recovery from $\mathrm{SCl}$.

\section{Introduction}

Spinal cord injury (SCl) is a damaging condition that might bring about irreversible disability. It is typically considered that $\mathrm{SCl}$ triggers an array of molecular and cellular events to cause neuronal injuries [1-3]. Amongst the factors of neuronal injuries, inflammation is a significant element in SCl pathogenesis [4-6]. Glia is an essential part of the nerves and is associated with the uptake of neurotransmitters, signal transduction, neurodevelopment, myelin synthesis, as well as immune reactions. Exosomes are vesicles in the extracellular space that are initially produced from particular kinds of cells. Exosomes are recognized to moderate glia activity. Glia-derived exosomes (GDEs) could move proteins, nucleotides as well as other cellular components among different kinds of cells. GDEs were shown to play both harmful and protective roles in the nerve system. The protective roles of GDEs have actually been shown in $\mathrm{SCl}$, TBI as well as ischemic stroke models [7-9]. 
As a major part of ncRNA with a size of about 22 nucleotides, microRNAs (miRNAs) play essential posttranscriptional roles in gene regulation by binding to 3-UTRs of target mRNAs [10-12]. MiRNAs also play essential functions in tumorigenesis $[13,14]$. Exosomes are tiny vesicles of $<100 \mathrm{~nm}$ in dimension and are produced by many types of cells [15]. Exosomes can carry microRNAs, mRNAs, IncRNAs, as well as proteins, thus playing vital functions in inducing certain activities in recipient cells, such as suppressing the activities of immune cells during cancer development $[16,17]$. Surprisingly, hypoxia can change the cargo of exosomes to affect cell-cell interactions $[18,19]$. The evaluation of microRNA sequences showed miR-1246 as one of the most abundant microRNAs in HGDEs. Furthermore, miR-1246 expression was enhanced in the cerebrospinal liquid (CSF) of patients suffering from GBM. Additional results showed that miR-1246 moderated the polarization of M2 macrophages by activating the STAT3 pathway while inhibiting the NF-KB pathway. In addition, miR-1246 expression was enhanced in the CSF of GBM patients but showed a substantial reduction after resection of tumors.

TERF2IP can regulate the functions of telomeres. Furthermore, TERF2IP can control the NF-KB pathway by enhancing IKK-mediated p65phosphorylation, thus increasing the expression of target genes of NF-KB. It was shown that miR-1246 may suppress TERF2IP expression to promote M2 polarization of macrophages by triggering the STAT3 signaling as well as suppressing the NF-KB signaling, consequently promoting the malignant development of glioma cells. It was shown that the suppression of TERF2IP expression may upregulate STAT3 expression and STAT3 phosphorylation, an essential factor in M2 polarization of macrophages [20]. M2 macrophages have the properties to modulate immune responses, promote tissue repair, and express molecular markers including arginase-1 as well as CD206 [21-23]. Previous researches have actually revealed that both $\mathrm{M} 2$ and $\mathrm{M} 1$ macrophages exist in the damaged spine, where the polarization of cytotoxic M1 macrophages is favored [24]. Since M2 macrophages can enhance axonal re-growth, raising the number of $\mathrm{M} 2$ cells can be an encouraging approach for post-SCI recovery [25-27].

Hypoxic GLIOMA derived exosomes may induce M2 macrophage polarization by upregulating TERF2IP expression [28]. Furthermore, M2 macrophage polarization was found to be associated with accelerated $\mathrm{SCl}$ recovery by suppressing inflammatory response [29]. In this study, GLIOMA cells were pre-treated with hypoxia before exosomes were isolated from the cells to treat a rat model of $\mathrm{SCl}$, so as to study the effect of hypoxic GLIOMA derived exosomes on $\mathrm{SCl}$ recovery and macrophage polarization.

\section{Materials And Methods}

\section{Animal and treatment}

Rats were purchased from our animal center and divided into 4 groups, i.e., 1. SHAM group (rats treated with sham operations); 2. SCl group (rats treated with operations to induce SCI); 3. SCI + U251-NG-EXO (rats treated with operations to induce $\mathrm{SCl}$ and then treated with exosomes isolated from U251 cells exposed to the NG condition); and 4. SCI + U251-HG-EXO (rats treated with operations to induce SCl and then treated with exosomes isolated from $\mathrm{U} 251$ cells exposed to the $\mathrm{HG}$ condition). In SHAM, SCI and SCI 
+ U251-NG-EXO groups, the U251 cells were cultured under the normal level of oxygen $\left(5 \% \mathrm{CO}_{2}\right.$ and $95 \%$

air) before the exosomes were isolated from cells for SCI treatment. In the SCI + U251-HG-EXO group, the U251 cells were cultured under a hypoxia condition before the exosomes were isolated from cells for SCl treatment.

\section{Surgical procedure to induce SCI}

Rats were arbitrarily designated into the above 4 groups to various treatments. In the sham group, the rats underwent the laminectomy procedure without inducing $\mathrm{SCl}$. In the SCI group, the rats were exposed to hemisection on the lateral side of the spinal cord. After the surgical procedure, rats were given postoperating treatment consisting of bowel as well as bladder treatment to avoid infection.

\section{Survival rate and BBB test}

Throughout the very first 21 day after the onset of SCl, the survival rate was determined according to the rate of fatality in each group. Then, the 21 point Basso-Beattie-Bresnahan (BBB) score was rated to analyze the locomotor activity of hind limbs in the rats prior to the procedure as well as in 8 weeks (once a week) following the onset of $\mathrm{SCl}$. In each assessment, the rats were put into an open area before they were observed for 5 minutes of independent movement. The BBB rating, which varied from 0 to 21 points, was evaluated blindly by 2 observers separately.

\section{RNA isolation and real-time PCR}

Total RNA content was isolated from samples by making use of an EZ-RNA II isolation assay kit (Biological Industries, Beit Haemek, Israel) according to the manual instructions provided by the supplier. The RNA samples were converted into cDNA by making use of a miScript Reverse Transcription assay kit (QIAGEN, Hilden, Germany) according to the manual instructions provided by the supplier. Then, the cDNA samples were amplified by real time PCR to evaluate the relative expression of CD163, IL10, IL-1RA, TGFB1, CCL2, TNF-a, miR-1246, let-7b, and TERF2IP in each sample by utilizing a miScript SYBR Green PCR assay kit (QIAGEN, Hilden, Germany) according to the manual instructions provided by the supplier. The real time PCR was done by utilizing a Light Cycler 480 machine (Roche Applied Science, Mannheim, Germany) according to the manual instructions provided by the supplier.

\section{Cell culture and transfection}

U937 cells and human GBM U251 cells were acquired from the Shanghai Cell Bank of the Chinese Academy of Sciences (Shanghai, China) and maintained in DMEM (Thermo Fisher Scientific, Waltham, MA) supplemented by $10 \%$ of FBS (Gibco, Thermo Fisher Scientific, Waltham, MA) and proper antibiotics. Then, U937 cells were randomly divided into 3 groups, i.e., 1. PBS group (U937 cells treated with PBS); 2. U251-NG-EXO group (U937 cells treated with exosomes extracted from U251 cells cultured under an NG condition); and 3. U251-HG-EXO group (U937 cells treated with exosomes extracted from U251 cells cultured under an HG condition). The U937 cells in various groups were cultured according to specified 
conditions for $48 \mathrm{~h}$ before they were harvested for analyses. In another experiments, U937 cells were randomly divided into 4 groups, i.e., 1. Scramble control group (U937 cells transfected with a scramble control); 2. miR-1246 precursor group (U937 cells transfected with miR-1246 precursors); 3 . let-7b precursor group (U937 cells transfected with let-7b precursors); and 4. group of miR-1246 + let-7b precursors (U937 cells transfected with let-7b and miR-1246 precursors). The transfection was carried out using Lipofectamine 2000 (Invitrogen, Carlsbad, CA) according to the manual instructions provided by the supplier. For co-culture with exosomes, $1 \mu \mathrm{g} / \mathrm{ml}$ of exosomes was suspended in the culture medium before cell culture was started.

\section{Exosome isolation}

Cells were incubated in DMEM for $48 \mathrm{~h}$ under conditions of normoxia $(21 \% 02)$ or hypoxia $(1 \% 02)$. Then, exosomes were separated from the cell supernatant using a routine method.

\section{Exosome evaluation by electron microscopy}

Cells were rinsed in PBS and prepared into a monolayer fixed in a pH 7.2 buffer containing $0.05 \mathrm{M}$ of cacodylate and $0.2 \%$ of glutaraldehyde. Then, the cells were dyed in uranyl acetate and observed underneath a $2100 \mathrm{~F}$ transmission electron microscope according to the manual instructions provided by the supplier.

\section{Detection of proportion of CD11b and CD163 positive cells by flow cytometry}

To quantify the proportion of CD11b and CD163 positive cells, cultured cells were treated with a staining kit (Thermo Fisher Scientific, Waltham, MA) and examined by a FACScan flow cytometer (BD, San Jose, $\mathrm{CA}$ ) in conjunction with CellQuest software according to the manual instructions provided by the supplier.

\section{Vector construction, mutagenesis and luciferase assay}

To explore the effect of U251-HG-EXO on the expression of miR-1246/let-7b and TERF2IP, binding site screening was performed for miR-1246 and let-7b, and the results identified TERF2IP as a potential target of miR-1246 and let-7b. Then, the 3' UTR sequences of TERF2IP containing the binding sites for miR-1246 and let-7b were inserted into pcDNA 3.1 luciferase vectors (Promega, Madison, WI) to generate wild type plasmids of TERF2IP for miR-1246 and let-7b, respectively. Then, single mutations were generated in the miR-1246 and let-7b binding sites of TERF2IP by using a Quick Change site directed mutagenesis assay kit (Stratagene, San Diego, CA)

according to the manual instructions provided by the supplier. The mutant sequences were also inserted into pcDNA 3.1 luciferase vectors to generate mutant type plasmids of TERF2IP for miR-1246 and let-7b, respectively. Then, U937 cells were co-transfected with wild type/mutant type plasmids of TERF2IP in conjunction with miR-1246 or let-7b mimics, respectively, by using Lipofectamine 2000 . The luciferase expression in transfected cells was assayed $48 \mathrm{~h}$ later by making use of a Dual-Glo luciferase reporter assay (Promega, Madison, WI) according to the manual instructions provided by the supplier. 


\section{Western blot evaluation}

Cell and tissue samples were lysed in a RIPA buffer before $20 \mu \mathrm{g}$ of protein in each sample were resolved by $12 \%$ NuPAGE Bis-Tris gel electrophoresis (Invitrogen, Carlsbad, CA). After the resolved proteins were transferred onto nitrocellulose membranes, the membranes were treated with $5 \%$ skim milk and then incubated in succession with anti-CD9, anti-CD61, and anti-TERF2IP primary antibodies as well as suitable secondary antibodies. Finally, the relative expression of CD9, CD61, and TERF2IP proteins was measured by using an ECL reagent (Thermo Fisher Scientific, Waltham, MA) according to the manual instructions provided by the supplier.

\section{Immunohistochemistry}

Samples were fixed for 20 minutes at ambient temperature in $4 \%$ paraformaldehyde, blocked in PBS containing $0.5 \%$ Triton X-100 and 5\% FCS, and incubated in succession with anti-TERF2IP primary antibody and a suitable secondary antibody. After counter staining with DAPI, the cells were visualized under a confocal microscope (Fluoview-FV-1000, Olympus, Tokyo, Japan) according to the manual instructions provided by the supplier to determine the relative protein expression of TERF2IP in each sample.

\section{ELISA}

Commercial kits (Thermo Fisher Scientific, Waltham, MA) were used according to the manual instructions provided by the supplier to measure the contents of IL-10 and TNFa in the CSF of SCl rats. The values of optical density (OD) were determined on a UVmax microplate reader (Molecular Devices, Sunnyvale, CA).

\section{TUNEL assay}

An Apo-BrdU In Situ DNA-Fragmentation assay kit (BioVision, Milpitas, CA) and an In Situ Cell Death Detection Fluorescein assay kit (Roche Diagnostics, Rotkreuz, Switzerland) were used according to the manual instructions provided by the suppliers to determine the status of apoptosis of collected spinal cord samples.

\section{Statistical analysis}

Inter-group differences were analyzed with Student's t tests and One-Way ANOVA using SPSS statistics software (SPSS, IBM, Chicago, IL). All results were expressed in mean \pm standard deviations. The degree of statistical significance was set to $5 \%$.

\section{Results}

Hypoxic U251 derived exosomes significantly altered the expression of CD163, IL-10, IL-1RA, TGFB1, CCL2 and TNFA in U937 cells. 
U251 cells were cultured under hypoxia or normal conditions followed by exosomes isolation. U937 cells were treated with exosomes isolated from U251 cells treated under either nomoxia (U251-NG-EXO) or hypoxia (U251-HG-EXO) conditions (Fig.1A). Western blot was performed to detect the CD9 and CD61 expression in the 3 groups. No obvious difference was detected for the expression of CD9 and CD61 among the PBS, U251-NG-EXO and U251-HG-EXO groups (Fig.1B). Then, the expression of CD163, IL-10, IL-1RA, TGFB1, CCL2 and TNFA mRNA was analyzed using qPCR in U937 cells treated with PBS, U251NG-EXO and U251-HG-EXO. The expression of CD163 (Fig.1C), IL-10 (Fig.1D), IL-1RA (Fig.1E), TGFB1 (Fig.1F) and CCL2 (Fig.1G) mRNA was progressively elevated in U937 cells treated with U251-NG-EXO and U251-HG-EXO. However, the expression of TNFA (Fig.1H) was gradually suppressed in U937 cells treated with U251-NG-EXO and U251-HG-EXO.

\section{Hypoxic U251 derived exosomes remarkably changed the proportions of CD11b+/CD163+ cells and IL- 10/TNF-a expression in U937 cells.}

Flow cytometry was performed to compare the proportions of CD11b+ and CD163+ U937 cells after treatment with U251-NG-EXO and U251-HG-EXO. U251-NG-EXO showed limited capacity to enhance the proportions of CD11b+/CD163+ cells, whereas U251-HG-EXO significantly increased the proportions of CD11b+/CD163+ cells (Fig.2A). Next, ELISA was carried out to quantify the levels of secreted IL-10 and TNF-a in the supernatant of U937 cells treated with U251-NG-EXO and U251-HG-EXO. The concentration of IL-10 was gradually increased in the supernatant of U937 cells treated with U251-NG-EXO and U251HG-EXO (Fig.2B). However, the concentration of TNF-a was gradually reduced in the supernatant of U937 cells treated with U251-NG-EXO and U251-HG-EXO (Fig.2C).

\section{Hypoxic U251 derived exosomes apparently promoted the expression of miR-1246 and suppressed the expression of TERF2IP in U937 cells.}

The expression of miR-1246 and let-7b was quantified in U937 cells treated under different conditions. U251-NG-EXO and U251-HG-EXO progressively reduced the expression of miR-1246 in U937 cells (Fig.3A). U251-NG-EXO showed no effect on the expression of let-7b in U937 cells, but U251-HG-EXO significantly up-regulated the expression of let-7b in U937 cells (Fig.3B). Meanwhile, the expression of TERF2IP mRNA and protein was examined in U937 cells treated under different conditions. The expression of TERF2IP in U937 cells was significantly inhibited by U251-NG-EXO and U251-HG-EXO in a progressive manner (Fig.3C).

\section{MiR-1246 and let-7b suppressed the expression of TERF2IP through binding to its 3' UTR.}

In order to further explore the effect of U251-HG-EXO on the expression of miR-1246/let-7b and TERF2IP, binding site screening was performed for miR-1246 and let-7b, and the results indicated that TERF2IP was a potential binding target of miR-1246 (Fig.4A) and let-7b (Fig.4C). Luciferase vectors containing wild type and mutant TERF2IP were established and co-transfected with miR-1246 and let-7b into U937 cells. The luciferase activities of wild type TERF2IP were remarkably repressed by miR-1246 (Fig.4B) and let-7b (Fig.4D). Moreover, miR-1246 precursor and let-7b precursor effectively suppressed the expression 
of TERF2IP in U937 cells. It was worth noting that simultaneous transfection of miR-1246 and let-7b precursors suppressed the expression of TERF2IP more efficiently (Fig.4E).

\section{Hypoxic U251 derived exosomes attenuated the spinal cord injury in SCl rats.}

Then, an SCl (spinal cord injury) rat model was established as described and treated with U251-NG-EXO and U251-HG-EXO to evaluate their therapeutic effects using a survival analysis and BBB scoring of the left hindlimb. U251-NG-EXO showed a moderate effect on improving the survival and BBB scores of left hindlimb of SCI rats. U251-HG-EXO significantly elevated the survival (Fig.5A) and BBB scores of left hindlimb (Fig.5B) of SCI rats. ELISA was used to measure the levels of IL-10 and TNF-a in the CSF of SCI rats. U251-NG-EXO and U251-HG-EXO progressively increased the level of IL-10 in the CSF of SCI rats (Fig.5C). However, the expression of TNF-a in the CSF was gradually suppressed by U251-NG-EXO and U251-HG-EXO (Fig.5D).

\section{Hypoxic U251 derived exosomes restored the normal expression of miR-1246, let-7b and TERF2IP in SCI rats.}

Quantitative real-time PCR was performed to analyze the differential expression of miR-1246, let-7b and TERF2IP in SCl rats treated under different conditions. The expression of miR-1246 (Fig.6A) and let-7b (Fig.6B) was effectively increased by U251-HG-EXO, whereas the expression of TERF2IP (Fig.6C) was repressed by U251-HG-EXO in SCl rats. Furthermore, the immunohistochemistry analysis showed that the expression of TERF2IP proteins in SCl rats was notably inhibited by U251-HG-EXO (Fig.7).

\section{Hypoxic U251 derived exosomes suppressed the apoptosis of spinal cord cells in SCl rats.}

As shown in Fig.8, TUNEL was carried out to evaluate the apoptosis of spinal cord cells in SCl rats treated under different conditions. U251-NG-EXO treatment showed considerable effects on decreasing the apoptosis of spinal cord cells in SCI rats. Furthermore, U251-HG-EXO treatment remarkably attenuated the apoptosis of spinal cord cells in SCl rats to a degree lower than that in the U251-NG-EXO group.

\section{Discussion}

In this study, we established a SCl rat model to evaluate the therapeutic effects of U251-NG-EXO and U251-HG-EXO, which progressively enhanced the survival and functional recovery of left hindlimb. Furthermore, U251-NG-EXO and U251-HG-EXO treatments promoted the expression of IL-10 and repressed the expression of TNFa in the CSF of SCl rats. In addition, we examined the expression of miR-1246, let7b and TERF2IP in the spinal cord of SCI rats. U251-NG-EXO and U251-HG-EXO treatments effectively restored the normal expression of miR-1246, let-7b and TERF2IP in SCI rats. Furthermore, we carried out a TUNEL assay to evaluate the apoptosis of spinal cord cells in SCI rats. U251-NG-EXO and U251-HG-EXO progressively attenuated the apoptosis of spinal cord cells in SCl rats. The protective effect of EXOs derived from various stem cells has been reported previously as discussed in the following paragraph 
and what's novel in this study is that we found that hypoxia exposure may further enhance such effect as well as its molecular mechanism underlying.

Previous study showed that GDEs enhance the interaction between glias and neurons while promoting the outgrowth of neuritis. As a result, GDEs may be associated with neurological conditions including glioblastoma, glioma, Parkinson's disease as well as Alzheimer's disease. GDE was also shown to play a role in MCAO, SCI, SNI, 5XFAD, GBM as well as TBI, highlighting the value of GDEs in the treatment of spine injury [7-9, 30]. Lately, numerous researches have actually shown that exosomes stemmed from BMSCs (BMSCs-Exos) may lower the apoptosis of neurons, reduce inflammatory reactions as well as enhance the recovery from SCI [31]. Another research revealed that exosomes from neuron cells increase the injury to BMSCs transplanted after $\mathrm{SCl}$, showing a prospective role of exosomes in reduced survival of transplanted BMSCs. Additionally, it was revealed that hypoxic pre-conditioning boosts BMSC survival. In addition, HIF-1 a plays a vital function in BMSC survival under oxidative stress. In this study, we isolated exosomes from U251 cells pretreated with nomoxia and hypoxia, and used the exosomes to treat U937 cells to explore their effects on the expression of CD163, IL-10, IL-1RA, TGFB1, CCL2 and TNFA. The expression of CD163, IL-10, IL-1RA, TGFB1 and CCL2 was gradually enhanced by U251-NG-EXO and U251-HG-EXO, while the expression of TNFA was progressively suppressed by U251-NG-EXO and U251HG-EXO. In addition, we performed flow cytometry to examine the proportions of CD11b+/CD163+ cells and carried out ELISA to analyze the concentrations of IL10 and TNFa in the supernatant of U937 culture medium. U251-NG-EXO and U251-HG-EXO progressively increased the proportions of CD11b+/CD163+ cells and IL10 concentration and decreased the concentration of TNFa. In line with previous studies, the protective effect of GDEs has been confirmed by the result of this study, and we further investigated the molecular mechanism underlying such effect which is its ability to suppress the inflammatory response following the injury via modulating the macrophage polarization.

Compared to normoxic GDEs (N-GDEs), hypoxic GDEs (H-GDEs) significantly triggered the polarization of M2 macrophages, consequently enhancing the migration, expansion, as well as invasion of glioma cells. Additionally, hypoxia can enhance the polarization of TAM M2 [32]. Past researches have actually shown that hypoxia could change the secretion of exosomes while affecting the features of recipient cells by controlling cell-cell interactions $[18,19,33,34]$. It was shown that hypoxia could influence the cargo of GDEs while increasing the immunosuppressive activities of myeloid-derived suppressor cells (MDSCs) $[19,35]$. MiR-1246 isolated from tumor cells was shown to enhance the polarization of M2 macrophages in individuals with glioma and promoted the development of GBMs. In this study, we measured the expression of miR-1246, let-7b and TERF2IP in U937 cells treated with U251-NG-EXO and U251-HG-EXO, which gradually elevated the expression of miR-1246 and decreased the expression of TERF2IP. U251-NGEXO had no effect on the expression of let-7b, but U251-HG-EXO remarkably activated the expression of let-7b. The results of this study from in vitro as well as in vivo identified the miRNAs and focus gene, TERF2IP, to modulate the macrophage polarization as the signaling pathway to mediate the function for inflammation suppression and recovery following the SCl. 
It was figured out that miR-1246 moderated the M2 polarization of macrophages by triggering the STAT3 pathway while suppressing the NF-KB pathway. For this reason, TERF2IP was assumed to be targeted by miR-1246 in the M2 polarization of macrophages. Indeed, TERF2IP was suppressed by the overexpression of miR-1246 and up-regulated by the inhibition of miR-1246. Additionally, the over-expression of TERF2IP can be reduced by miR-1246 mimics. Meanwhile, we performed luciferase assays to explore the regulatory role of miR-1246 and let-7b in the expression of TERF2IP. MiR-1246 and let-7b effectively suppressed the expression of TERF2IP in U937 cells.

Previous research revealed that TERF2IP over-expression inhibited the effect of miR-1246 on the M2 polarization of macrophages. These data suggested that miR-1246 caused the M2 polarization of macrophages by targeting TERF2IP. Taken together, these data indicated that H-GDE-derived miR-1246 reduced the expression of TERF2IP to trigger STAT3 pathway while suppressing NF-KB pathway in the macrophage, hence inducing the M2 polarization of macrophages [36]. As a member of the protein complex Shelterin specific to telomeres, TERF2IP plays a vital role in suppressing the repair of chromosomal breaks in telomeres [37]. The level of PTEN expression is considerably boosted in macrophages of SCI patients. In the culture of macrophages, the inhibition of PTEN expression or the inhibition in PTEN phosphorylation enhanced the M2 polarization of macrophages while reducing the M1 polarization of macrophages. As reported previously, the increase in the number of M2 macrophages at the site of SCl injury enhanced the outgrowth of axons to promote tissue repair and healing of $\mathrm{SCl}$ [27]. Our study confirmed the role of TERF2IP in the development of inflammation and regulation of macrophage polarization. Furthermore, we also identified the regulator of this gene as well as the effect of whole signaling pathway in the control of recovery of SCl. There is limitation of this study, there are only animal study and cellular study in this study and further study in human will further confirm or deny the protective effect of H-GDEs or N-GDEs in the recovery of SCl. In addition, further study with more comprehensive network involving more genes and signaling is needed.

\section{Conclusion}

The findings of this study demonstrated that glioma derived exosomes upregulated the expression of miR-1246 and let-7b to suppress the expression of TERF2IP to induce M2 macrophage polarization. The promoted M2 macrophage polarization suppressed inflammatory response to accelerate the recovery from SCl.

\section{Abbreviation}

SCl: spinal cord injury

EXO: exosomes

HG: hypoxia

NG: normoxia 
GDEs: glioma derived exosomes

\section{Declarations}

\section{Acknowledgement}

This study was sponsored by the academic subject boosting plan in the Shanghai Fourth People's Hospital affiliated to Tongji University School of Medicine (Grant No. SY-XKZT-2019-1004) and the academic general project from the Health commission of Hongkou District,Shanghai (Grant No. 1902-10).

\section{Conflict of interest}

None

\section{Availability of data and material}

The data of this study are available from the corresponding author upon reasonable request.

\section{AUTHOR CONTRIBUTIONS}

GHX and FRW planned the study,ZZZ CLZ YJW and JK collected the literatures, collected and analyzed the data, WY and JDL composed the manuscript, and all co-authors approved the final manuscript.

\section{Ethics approval}

All animal experiments were performed in line with the Guide for the Care and Use of Laboratory Animal by International Committees and were approved by the Animal Ethics Committee of Shanghai Fourth People's Hospital affiliated to Tongji University School of Medicine. The study is reported in accordance with ARRIVE guidelines

\section{Consent for publication}

Not applicable

\section{References}

1. Choo AM, Liu J, Dvorak M, Tetzlaff W, Oxland TR: Secondary pathology following contusion, dislocation, and distraction spinal cord injuries. Exp Neurol 2008;212:490-506.

2. Kwon BK, Tetzlaff W, Grauer JN, Beiner J, Vaccaro AR: Pathophysiology and pharmacologic treatment of acute spinal cord injury. Spine J 2004;4:451-464.

3. Hausmann ON: Post-traumatic inflammation following spinal cord injury. Spinal Cord 2003;41:369378. 
4. Donnelly DJ, Popovich PG: Inflammation and its role in neuroprotection, axonal regeneration and functional recovery after spinal cord injury. Exp Neurol 2008;209:378-388.

5. Trivedi A, Olivas AD, Noble-Haeusslein LJ: Inflammation and Spinal Cord Injury: Infiltrating Leukocytes as Determinants of Injury and Repair Processes. Clin Neurosci Res 2006;6:283-292.

6. Chavarria A, Alcocer-Varela J: Is damage in central nervous system due to inflammation? Autoimmun Rev 2004;3:251-260.

7. Adolf A, Rohrbeck A, Munster-Wandowski A, Johansson M, Kuhn HG, Kopp MA, Brommer B, Schwab JM, Just I, Ahnert-Hilger G, Holtje M: Release of astroglial vimentin by extracellular vesicles: Modulation of binding and internalization of C3 transferase in astrocytes and neurons. Glia 2019;67:703-717.

8. Hira K, Ueno Y, Tanaka R, Miyamoto N, Yamashiro K, Inaba T, Urabe T, Okano H, Hattori N: AstrocyteDerived Exosomes Treated With a Semaphorin 3A Inhibitor Enhance Stroke Recovery via Prostaglandin D2 Synthase. Stroke 2018;49:2483-2494.

9. Huang S, Ge X, Yu J, Han Z, Yin Z, Li Y, Chen F, Wang H, Zhang J, Lei P: Increased miR-124-3p in microglial exosomes following traumatic brain injury inhibits neuronal inflammation and contributes to neurite outgrowth via their transfer into neurons. FASEB J 2018;32:512-528.

10. Mohr AM, Mott JL: Overview of microRNA biology. Semin Liver Dis 2015;35:3-11.

11. Bartel DP: MicroRNAs: genomics, biogenesis, mechanism, and function. Cell 2004;116:281-297.

12. Fabian MR, Sonenberg N, Filipowicz W: Regulation of mRNA translation and stability by microRNAs. Annu Rev Biochem 2010;79:351-379.

13. Ji ZC, Han SH, Xing YF: Overexpression of miR-3196 suppresses cell proliferation and induces cell apoptosis through targeting ERBB3 in breast cancer. Eur Rev Med Pharmacol Sci 2018;22:8383-8390.

14. Hosseinahli N, Aghapour M, Duijf PHG, Baradaran B: Treating cancer with microRNA replacement therapy: A literature review. J Cell Physiol 2018;233:5574-5588.

15. Thery C, Zitvogel L, Amigorena S: Exosomes: composition, biogenesis and function. Nat Rev Immunol 2002;2:569-579.

16. Tkach M, Thery C: Communication by Extracellular Vesicles: Where We Are and Where We Need to Go. Cell 2016;164:1226-1232.

17. lero $M$, Valenti R, Huber V, Filipazzi P, Parmiani G, Fais S, Rivoltini L: Tumour-released exosomes and their implications in cancer immunity. Cell Death Differ 2008;15:80-88.

18. King HW, Michael MZ, Gleadle JM: Hypoxic enhancement of exosome release by breast cancer cells. BMC Cancer 2012;12:421. 
19. Guo X, Qiu W, Liu Q, Qian M, Wang S, Zhang Z, Gao X, Chen Z, Xue H, Li G: Immunosuppressive effects of hypoxia-induced glioma exosomes through myeloid-derived suppressor cells via the miR10a/Rora and miR-21/Pten Pathways. Oncogene 2018;37:4239-4259.

20. Giurisato E, Xu Q, Lonardi S, Telfer B, Russo I, Pearson A, Finegan KG, Wang W, Wang J, Gray NS, Vermi W, Xia Z, Tournier C: Myeloid ERK5 deficiency suppresses tumor growth by blocking protumor macrophage polarization via STAT3 inhibition. Proc Natl Acad Sci U S A 2018;115:E2801-E2810.

21. Ding N, Wang Y, Dou C, Liu F, Guan G, Wei K, Yang J, Yang M, Tan J, Zeng W, Zhu C: Physalin D regulates macrophage M1/M2 polarization via the STAT1/6 pathway. J Cell Physiol 2019;234:8788-8796.

22. Mosser DM, Edwards JP: Exploring the full spectrum of macrophage activation. Nat Rev Immunol 2008;8:958-969.

23. Stout RD, Suttles J: Functional plasticity of macrophages: reversible adaptation to changing microenvironments. J Leukoc Biol 2004;76:509-513.

24. Gensel JC, Zhang B: Macrophage activation and its role in repair and pathology after spinal cord injury. Brain Res 2015;1619:1-11.

25. Kong X, Gao J: Macrophage polarization: a key event in the secondary phase of acute spinal cord injury. J Cell Mol Med 2017;21:941-954.

26. Ji XC, Dang YY, Gao HY, Wang ZT, Gao M, Yang Y, Zhang HT, Xu RX: Local Injection of Lenti-BDNF at the Lesion Site Promotes M2 Macrophage Polarization and Inhibits Inflammatory Response After Spinal Cord Injury in Mice. Cell Mol Neurobiol 2015;35:881-890.

27. Ma SF, Chen YJ, Zhang JX, Shen L, Wang R, Zhou JS, Hu JG, Lu HZ: Adoptive transfer of M2 macrophages promotes locomotor recovery in adult rats after spinal cord injury. Brain Behav Immun 2015;45:157-170.

28. Qian M, Wang S, Guo X, Wang J, Zhang Z, Qiu W, Gao X, Chen Z, Xu J, Zhao R, Xue H, Li G: Hypoxic glioma-derived exosomes deliver microRNA-1246 to induce M2 macrophage polarization by targeting TERF2IP via the STAT3 and NF-kappaB pathways. Oncogene 2019;10.1038/s41388-019-0996-y

29. Peng Z, Gao W, Yue B, Jiang J, Gu Y, Dai J, Chen L, Shi Q: Promotion of neurological recovery in rat spinal cord injury by mesenchymal stem cells loaded on nerve-guided collagen scaffold through increasing alternatively activated macrophage polarization. J Tissue Eng Regen Med 2018;12:e1725e1736.

30. Gosselin RD, Meylan P, Decosterd I: Extracellular microvesicles from astrocytes contain functional glutamate transporters: regulation by protein kinase $C$ and cell activation. Front Cell Neurosci 2013;7:251. 
31. Sakha S, Muramatsu T, Ueda K, Inazawa J: Exosomal microRNA miR-1246 induces cell motility and invasion through the regulation of DENND2D in oral squamous cell carcinoma. Sci Rep 2016;6:38750.

32. Guo X, Xue H, Shao Q, Wang J, Guo X, Chen X, Zhang J, Xu S, Li T, Zhang P, Gao X, Qiu W, Liu Q, Li G: Hypoxia promotes glioma-associated macrophage infiltration via periostin and subsequent $\mathrm{M} 2$ polarization by upregulating TGF-beta and M-CSFR. Oncotarget 2016;7:80521-80542.

33. Tadokoro H, Umezu T, Ohyashiki K, Hirano T, Ohyashiki JH: Exosomes derived from hypoxic leukemia cells enhance tube formation in endothelial cells. J Biol Chem 2013;288:34343-34351.

34. Li L, Li C, Wang S, Wang Z, Jiang J, Wang W, Li X, Chen J, Liu K, Li C, Zhu G: Exosomes Derived from Hypoxic Oral Squamous Cell Carcinoma Cells Deliver miR-21 to Normoxic Cells to Elicit a Prometastatic Phenotype. Cancer Res 2016;76:1770-1780.

35. Guo X, Qiu W, Wang J, Liu Q, Qian M, Wang S, Zhang Z, Gao X, Chen Z, Guo Q, Xu J, Xue H, Li G: Glioma exosomes mediate the expansion and function of myeloid-derived suppressor cells through microRNA-29a/Hbp1 and microRNA-92a/Prkar1a pathways. Int J Cancer 2019;144:3111-3126.

36. Kanichai M, Ferguson D, Prendergast PJ, Campbell VA: Hypoxia promotes chondrogenesis in rat mesenchymal stem cells: a role for AKT and hypoxia-inducible factor (HIF)-1alpha. J Cell Physiol 2008;216:708-715.

37. Li H, Jiang T, Li MQ, Zheng XL, Zhao GJ: Transcriptional Regulation of Macrophages Polarization by MicroRNAs. Front Immunol 2018;9:1175.

\section{Figures}

A

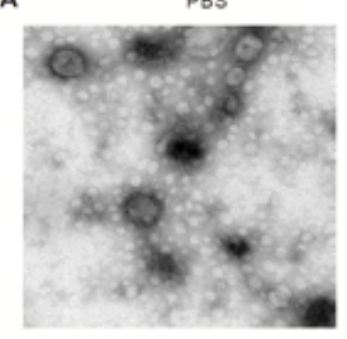

D

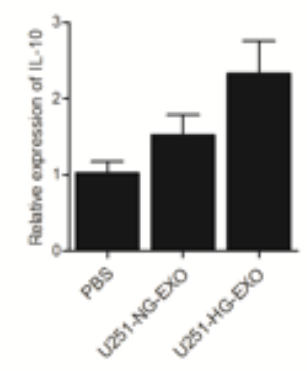

U251-NG-EXO

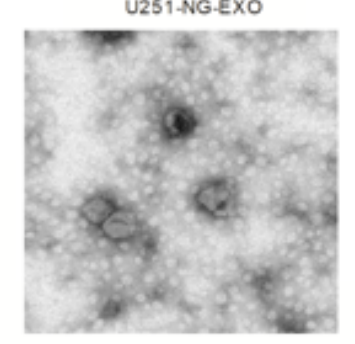

E

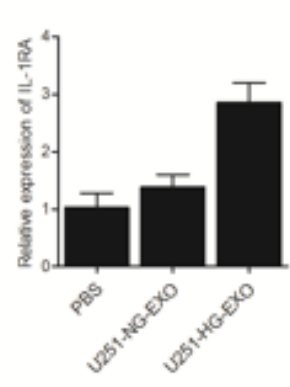

U251-HG-EXO

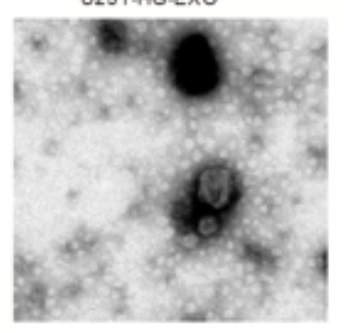

F

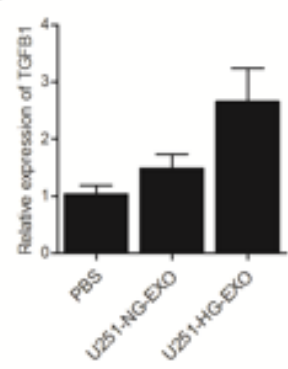

B

$\operatorname{CO} 9$

CD61

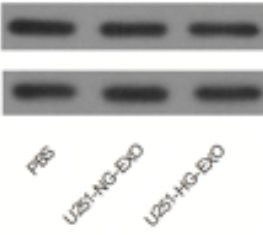

G

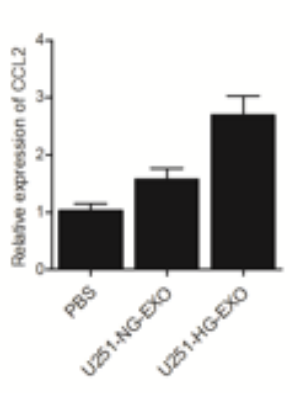

C

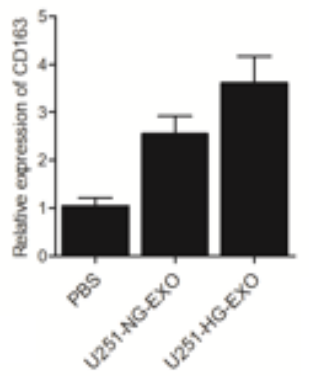

H

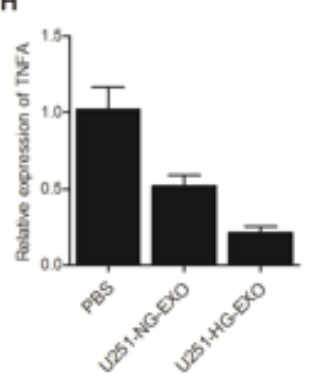


Figure 1

The expression of CD163, IL-10, IL-1RA, TGFB1, CCL2 and TNFA in U937 cells was altered by treatment with hypoxic U251 derived exosomes. A: Electron microscope image of exosomes derived from U251 cells treated under nomoxia and hypoxia conditions. B: Western blot showed CD9 and CD61 expression in exosomes. C: U251-NG-EXO and U251-HG-EXO progressively elevated the expression of CD163 in U937 cells. D: U251-NG-EXO and U251-HG-EXO progressively elevated the expression of IL10 in U937 cells. E: U251-NG-EXO and U251-HG-EXO progressively elevated the expression of IL-1RA in U937 cells. F: U251NG-EXO and U251-HG-EXO progressively elevated the expression of TGFB1 in U937 cells. G: U251-NGEXO and U251-HG-EXO progressively elevated the expression of CCL2 in U937 cells. H: U251-NG-EXO and U251-HG-EXO progressively decreased the expression of TNFA in U937 cells.

A

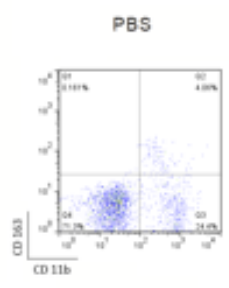

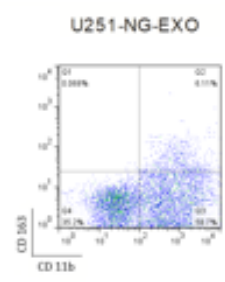

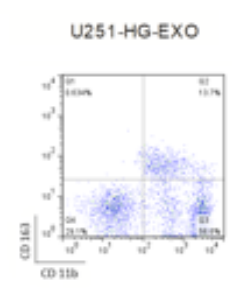

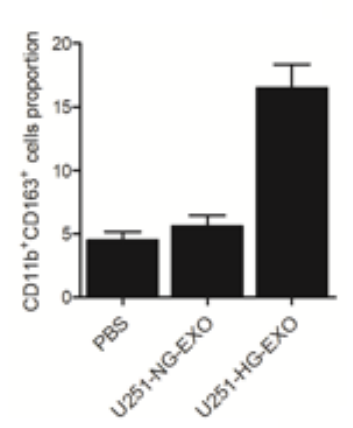

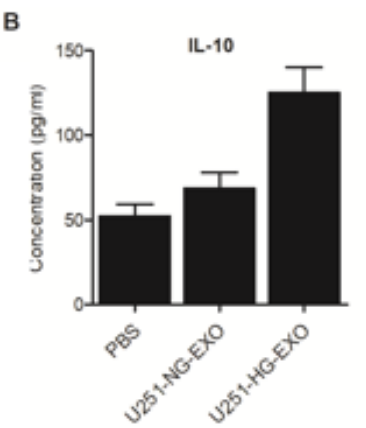

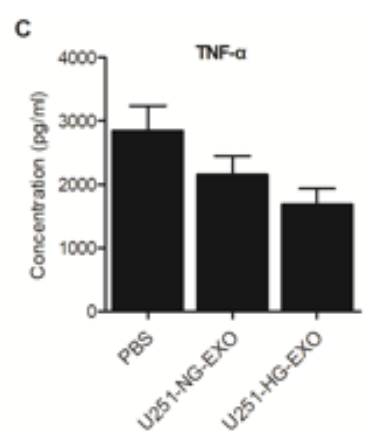

\section{Figure 2}

U251-NG-EXO and U251-HG-EXO treatments changed the proportion of CD11b+/CD163+ cells and the expression of IL-10 and TNFa in U937 cells. A: The proportion of CD11b+/CD163+ cells was increased by U251-NG-EXO and U251-HG-EXO treatments. B: The concentration of IL10 in the supernatant was elevated by U251-NG-EXO and U251-HG-EXO treatments. C: The concentration of TNFa in the supernatant was decreased by U251-NG-EXO and U251-HG-EXO treatments. 
A

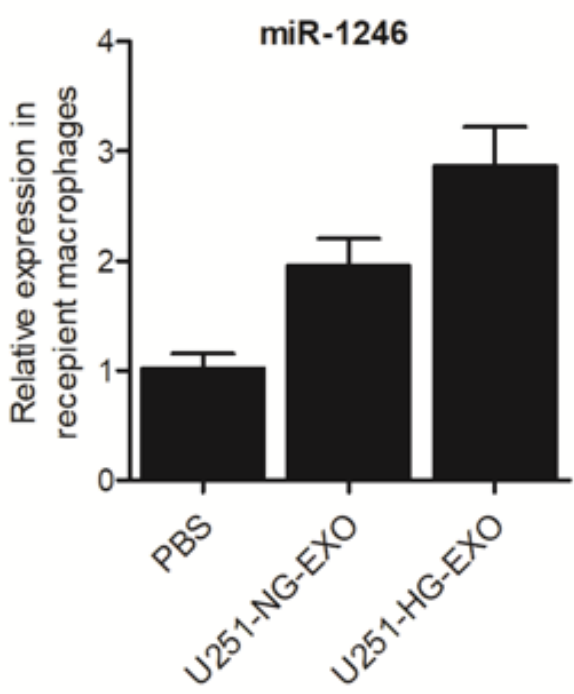

B

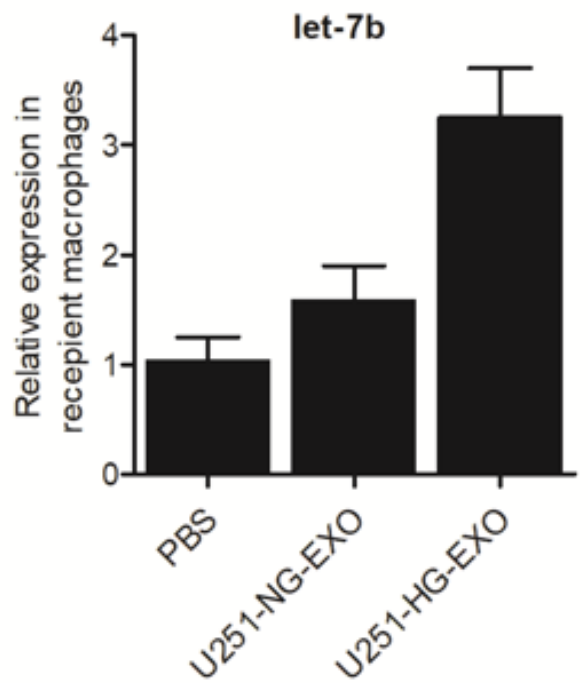

C TERF2IP

$\beta$-actin

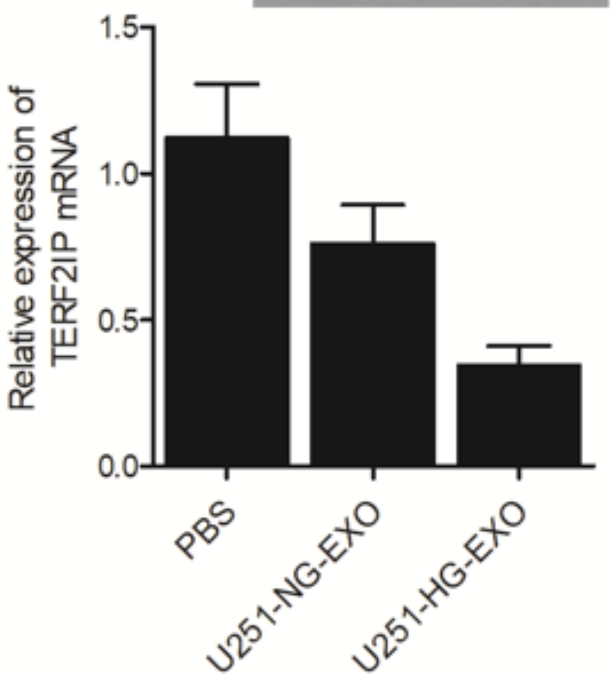

Figure 3

The expression of miR-1246, let-7b and TERF2IP was altered by U251-NG-EXO and U251-HG-EXO in U937 cells. A: The expression of miR-1246 was activated by U251-NG-EXO and U251-HG-EXO in U937 cells. B: The expression of let-7b was activated by U251-NG-EXO and U251-HG-EXO in U937 cells. C: The expression of TERF2IP was suppressed by U251-NG-EXO and U251-HG-EXO in U937 cells. 
A

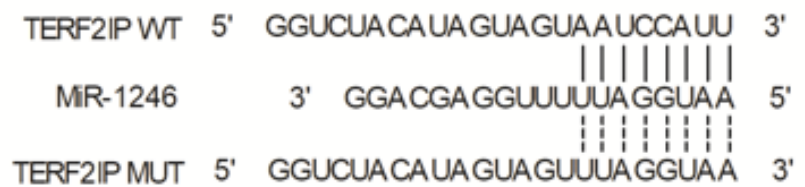

B
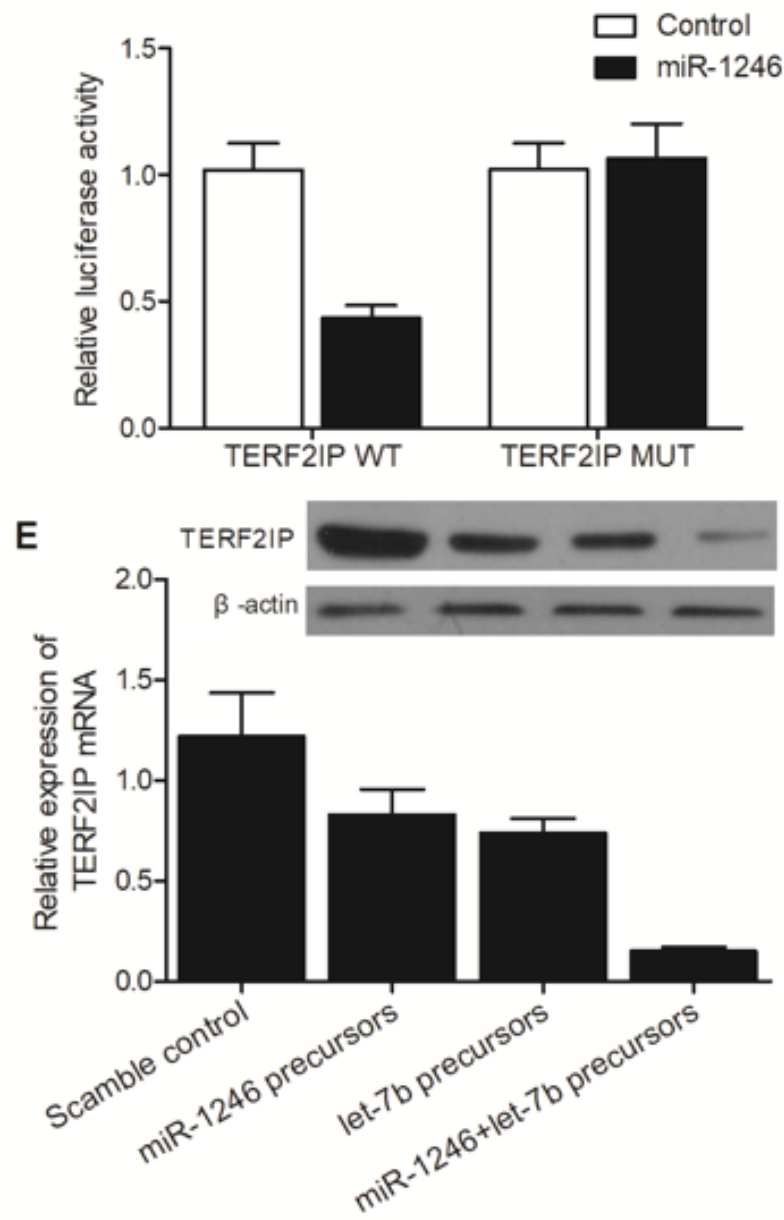

C

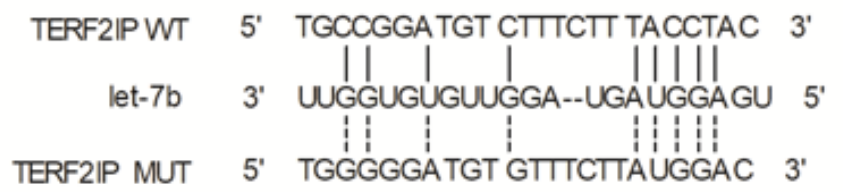

D

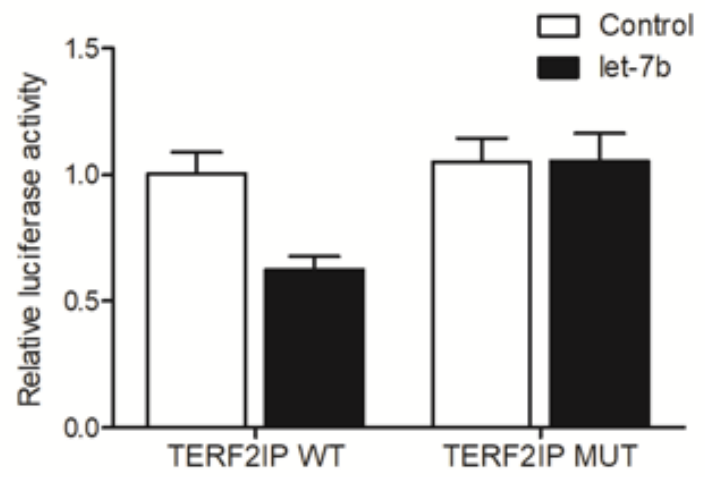

\section{Figure 4}

MiR-1246 and let-7b inhibited the expression of TERF2IP by binding to its 3' UTR. A: Sequence analysis showed potential binding of miR-1246 to TERF2IP. B: The luciferase activity of wild type TERF2IP was inhibited by miR-1246. C: Sequence analysis showed potential binding of let-7b to TERF2IP. D: The luciferase activity of wild type TERF2IP was inhibited by let-7b. E: MiR-1246 and let-7b precursors effectively inhibited the expression of TERF2IP in U937 cells. 

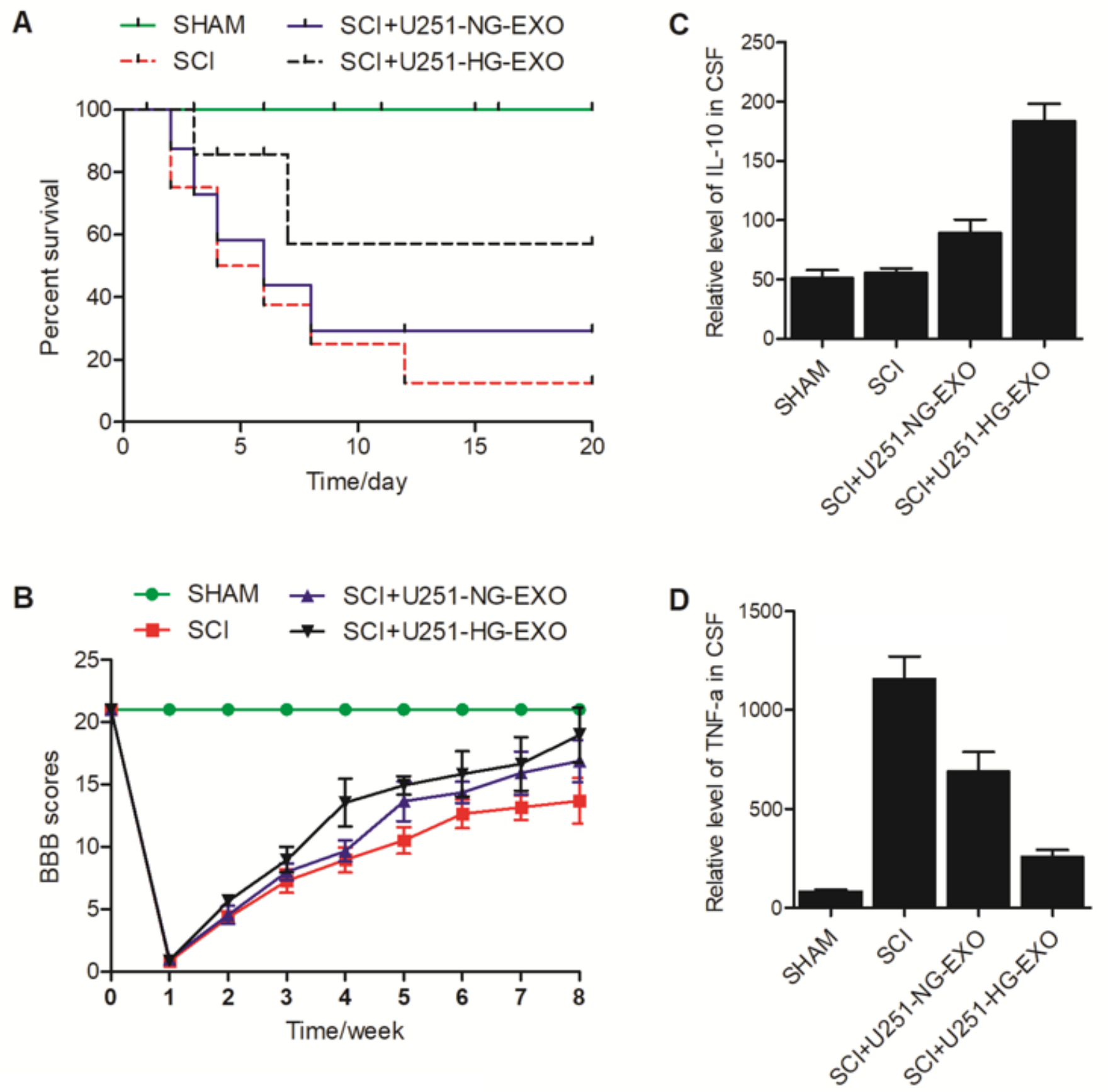

Figure 5

The survival and functional recovery of left hindlimb of SCI rats were promoted by U251-NG-EXO and U251-HG-EXO. A: The survival of SCI rats was elevated by U251-NG-EXO and U251-HG-EXO. B: The recovery of left hindlimb of SCI rats was elevated by U251-NG-EXO and U251-HG-EXO. C: U251-NG-EXO and U251-HG-EXO treatments effectively increased the expression of IL-10 in CSF of SCI rats. D: U251NG-EXO and U251-HG-EXO treatments effectively decreased the expression of IL-10 in CSF of SCI rats. 
A

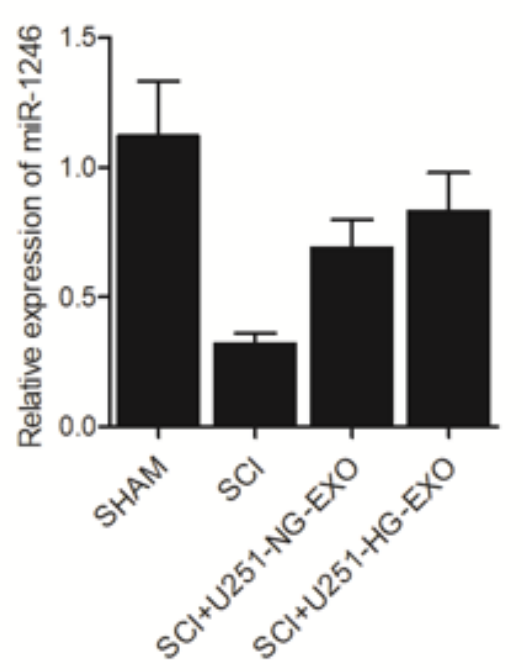

B

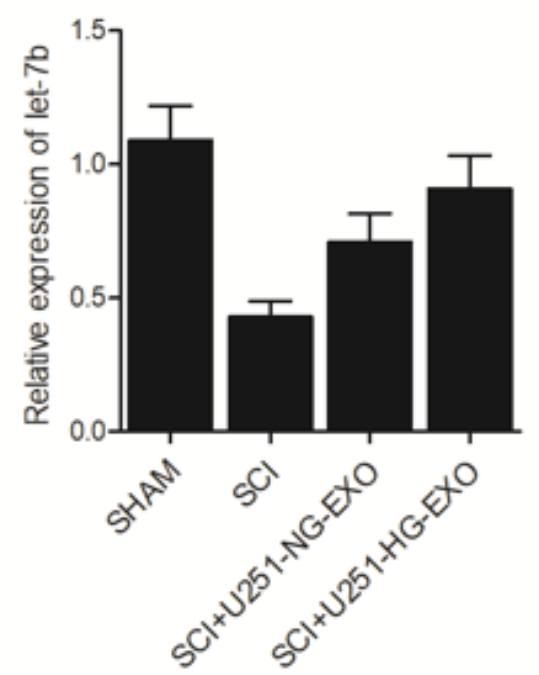

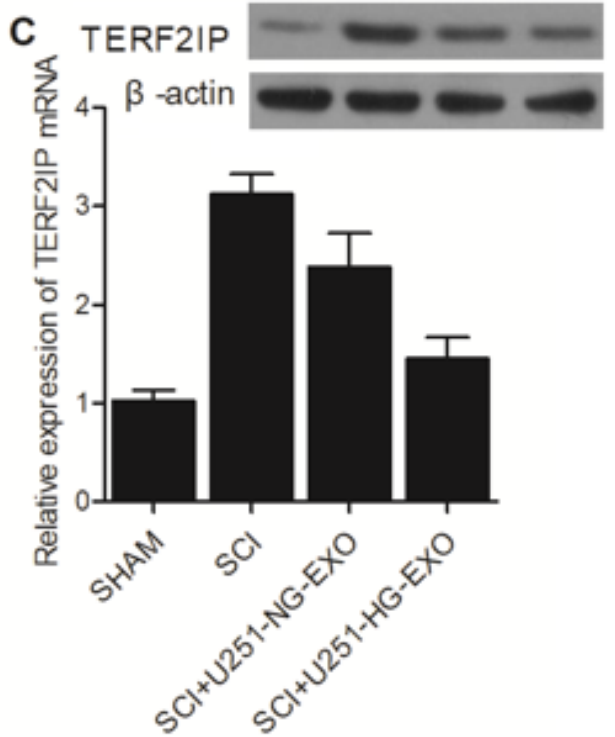

Figure 6

U251-NG-EXO and U251-HG-EXO treatments restored the expression of miR-1246, let-7b and TERF2IP in $\mathrm{SCl}$ rats. A: The expression of miR-1246 in SCl rats was restored by U251-NG-EXO and U251-HG-EXO treatments. B: The expression of let-7b in SCl rats was restored by U251-NG-EXO and U251-HG-EXO treatments. C: The expression of TERF2IP mRNA in SCI rats was restored by U251-NG-EXO and U251-HGEXO treatments.
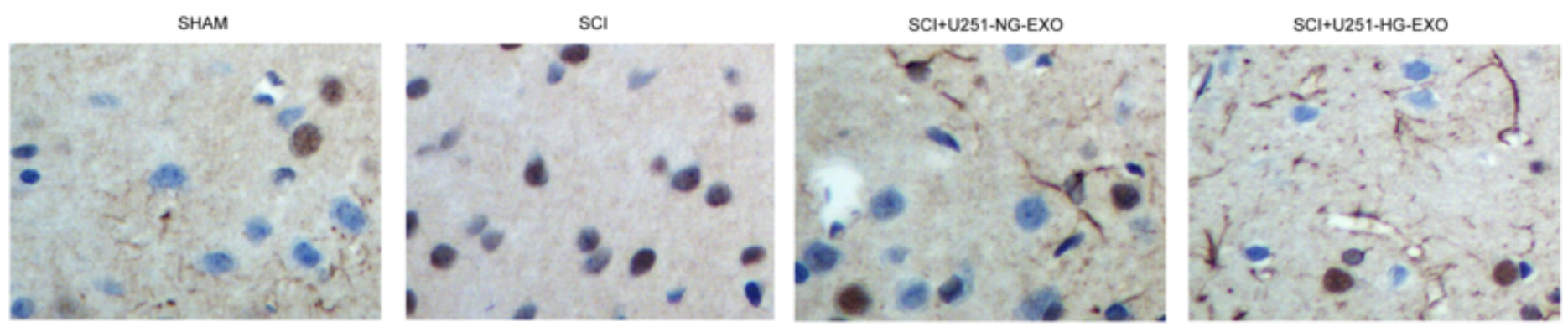

\section{Figure 7}

IHC analysis showed that the expression of TERF2IP protein in SCI rats was restored by U251-NG-EXO and U251-HG-EXO treatments.
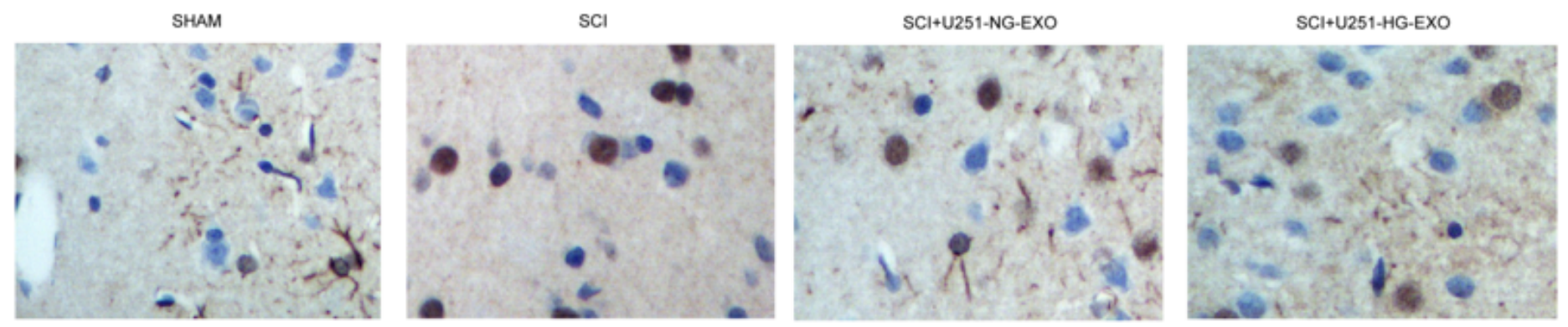
Figure 8

U251-NG-EXO and U251-HG-EXO treatments attenuated the apoptosis of spinal cord cells in SCI rats. 